\title{
Object Localization Based Segmentation and Spatial Analysis Using Computer Vision
}

\author{
Erkan Bostanci, Member, IACSIT and Betul Bostanci
}

\begin{abstract}
This paper extends the classical segmentation method known as region growing using a new localization method which uses algorithms from computer science and computer vision. Described method was successfully applied to the problem of cell localization and segmentation. The method proves to be useful as part of a whole autonomous segmentation process. Furthermore, methods from spatial statistics were employed in order to model the spatial distribution of the localized objects. In-depth discussion of these methods including density plots and the Ripley's $\mathrm{K}$ function is presented along with the results for test set used in the experiments.
\end{abstract}

Index Terms-Object localization, segmentation, spatial analysis

\section{INTRODUCTION}

In many computer vision applications, finding the position of an object is an important part of the process. Especially for object recognition, the objects in the image must be first isolated from the remaining objects or clutter in the scene. Then it is straight-forward to match these objects with the ones stored in an image database [1]. Literature presents several methods for this purpose. Clustering algorithms as in [2], [3] and segmentation methods similar to [4]-[6] are well-known techniques in this regard.

However, there are some issues which prevent current algorithms from being tools for an autonomous system. For instance, clustering algorithms require the number of clusters which will be generated as the output of the system [2]. Similarly, the segmentation methods generally require a seed point for the algorithm [5]. This need for human supervision obviously reduces the autonomy of the system if the intended systems are autonomous systems like robots.

Furthermore, finding and modelling a relationship between these detected objects is also an important issue in many cases. Spatial Data Analysis concerns drawing such relationships between objects based on their spatial properties i.e. positions, area coverage, density [7]. These types of applications are generally used in biological analysis of populations for different ecosystems at larger scales [8] or other GIS related studies [9].

This paper develops on [10], [11] and extends the classical region growing algorithm using automated object localization which is based on Minimum Spanning Trees and the convex-hull here. To our best knowledge, the closest approach to the method defined in this paper is presented in [12] where Minimum Spanning Trees are used for image

Manuscript received September 29, 2012; revised December 11, 2012.

The authors are with the University of Essex, Colchester, UK (e-mail: gebost@essex.ac.uk, bbosta@essex.ac.uk). mining. Our approach differs from them both for the purpose of usage and the method itself as our method adds the usage of feature points together with the convex-hull. In addition, this study also applies techniques from spatial statistics on the objects localized with the proposed method.

The rest of the paper is structured as follows: Section II describes the approach taken for localizing image objects. Segmentation approach is described in Section III and later in Section IV an experiment carried out as a test bed for this approach is presented. Section V describes the spatial analysis process carried out on the detected objects. Finally, findings of the paper are discussed and future research is presented in Section VI.

\section{LOCALIZATION METHOD}

The localization method proposed here uses Minimum Spanning Trees which are generally used for network routing in order to minimize the number of hops for packages [13]. Application to computer vision is new concept after the recent clustering algorithm for image mining [12]. There are two main algorithms for calculating the Minimum Spanning Tree of a given graph, namely Kruskal [14] and Prim [15].

The graph in our algorithm is constructed using the $2 \mathrm{D}$ positions of the point features obtained by the FAST operator [16] which is a state-of-the-art feature extraction method. The graph is in form of an adjacency matrix where the cells with nonzero values denote an edge between the corresponding vertices.

This graph is fully-connected as there will be edges between all vertices. The weights of the edges are the Euclidian distances between the features on the image.

The Minimum Spanning Tree of the graph is constructed using Prim's algorithm (This choice is arbitrary as both methods can be used here since the graph is fully-connected). Prim's algorithm calculates the Minimum Spanning Tree in $\mathrm{O}\left(\mathrm{E}^{*} \log (\mathrm{V})\right)$ time where $\mathrm{E}$ is the number of edges and $\mathrm{V}$ is the number of vertices. This minimal graph has the same format as the original graph that is the edges between the vertices still denote the distances.

These distances give clues about the positions of the image objects when we consider a specific region covering features points in the objects. This property is used as the localization approach in the algorithm with the assumption that the feature points of an object must lay within a known distance. When the edges with weights greater than this distance threshold are removed, the remaining edges will define the locations of individual groups along with the vertices.

The convex-hull of the remaining feature group is then calculated to define the outline of the localized object. The 
convex-hull itself presents an overestimation as it includes the features at the outer region only and do not consider the features in the inner region. However, the Minimum Spanning Tree approach used here prevents this overestimation and produces a better result.

The method used for localization of image objects can be summarized with the following steps:

1) Extraction of FAST point features.

2) Generation of a graph including these feature points where the vertices will denote the positions of point features and the weights of the edges will represent the distances between the features.

3) Computation of Minimum Spanning Tree.

4) Removal of edges greater than a predefined threshold.

5) Calculation of the convex-hull of the vertex groups based on the remaining graph.

Having defined the localization method, next sections will develop a segmentation method based on this localization method and later present a sample application showing how this method is successfully applied to the problem of biological cell localization.

\section{SEgmentATION}

Identifying feature points belonging to the same object is quite useful in many applications and the proposed localization method performs well on identifying data points belonging to the same object. However, better results can be achieved if this method can be extended to a segmentation method that is able to extract the boundary of an object. With this research question in mind, proposed method was further used in a conventional segmentation method known as region growing [17], [18].

Given an initial seed point, region growing algorithm determines whether to add neighboring pixels to the region or not based on a similarity criterion such as mean intensity. Selection of a good seed-point plays an important role in the segmentation process. In our method, we used the points which were found as the output of the localization algorithm and corresponding to individual objects as the seed points of the region growing algorithm given below:

Input: Image $I$, list of seed points $S$, threshold $T$

Output: Image $R$

1) Calculate the mean of current $R$ as $R_{m}$.

2) Define template $C$ to access the 4-neighbour pixels around the seed.

3) For all seed points in $S$

While the neighboring pixels values are close to the $R_{m}$ and there are pixels to process

- Calculate coordinates of neighbors using $C$. Check if a neighbor is outside the image boundary. Discard the neighbor if this is the case.

- Calculate the distance between $R_{m}$ and the neighbors' intensity. Take the neighbours with distance to $R_{m}$ less than $T$ but the neighbor with the minimum distance must be added to $R$.

- Update $\mathrm{R}_{\mathrm{m}}$ including the newly joint neighbour.

4) Return $R$

\section{ApPliCATION TO LOCALIZATION AND SEGMENTATION OF BiologicAl CELlS}

The proposed method was applied to the biological cell localization problem in order to improve the autonomy of the process. Following two subsections will describe the test data used for this purpose and the results of the application.

\section{A. Test Data}

The test data for experimentation was obtained from The Cell: An Image Library [19]. The images of fibroblast cells were used for this experiment as shown in Fig. 1 in the test culture they were developed.

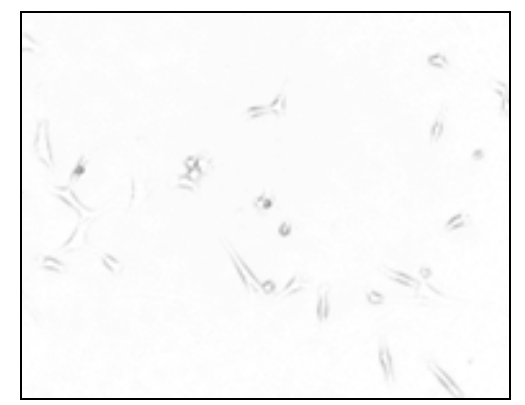

Fig. 1. Fibroblast cells of type NIH 3T3.

The original images included tif image sequences showing the biological process of localization. A detailed explanation about the whole process is presented along with each data set in the library.

\section{B. Localization and Segmentation of Cells}

The experiment carried out on the image datasets aims to identify individual cells as shown in Fig. 2 where the cells are placed in a special culture and some chemicals are used to present better imaging results.

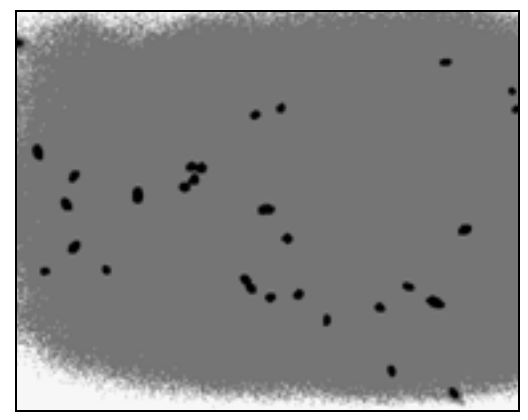

Fig. 2. Result of biological localization process (7826).

FAST operator was applied to the image in order to extract feature points. The operator selects the prominent point locations in the image corresponding to the cells. Result is presented in Fig. 3.

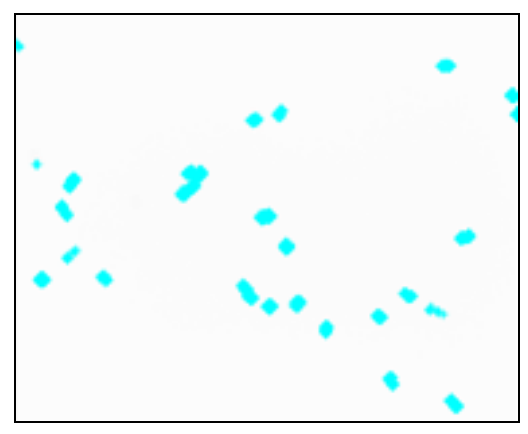

Fig. 3. Extracted FAST features. 
A graph is created by using the feature points though it is not shown here as it is very complex since it is fully-connected. The Minimum Spanning Tree is calculated as shown in Fig. 4.

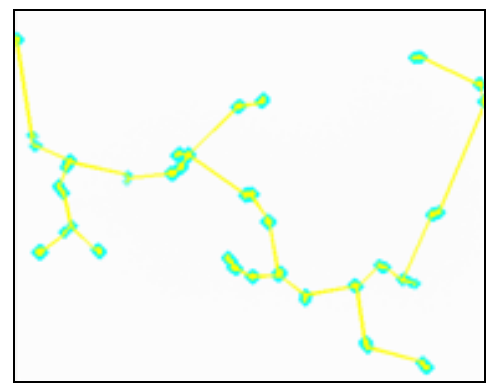

Fig. 4. Minimum spanning tree of the feature points.

The edges with a weight which is greater than a predefined threshold (in image pixels while microns are used to define these distances in the actual culture environment) are removed leaving many groups of feature points. These groups identify the positions of the cells in the image as shown in Fig. 5. The convex-hull was then used to extract the whole region including the cell.

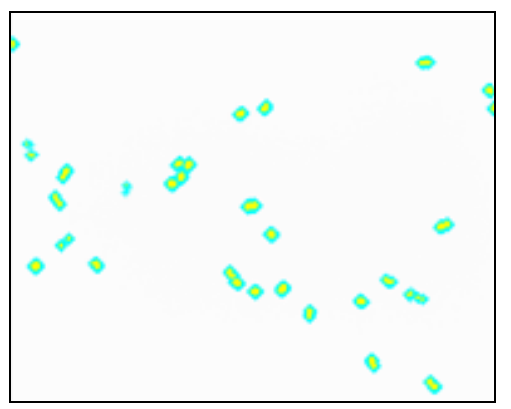

Fig. 5. Localized cells after removing longest edges.

The results of application to another test set are presented in Figs. 6(a) and 6(b) where localized cells are shown with blue marks in the centre.

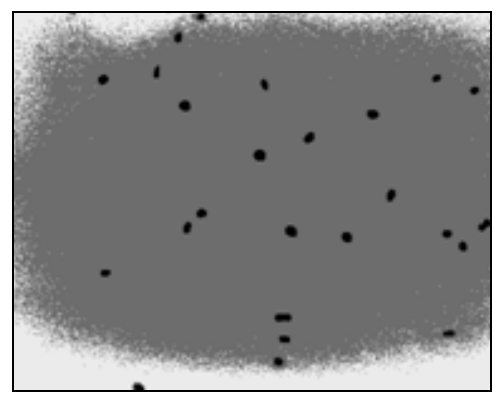

(a) A different set for the same cell type (8924)

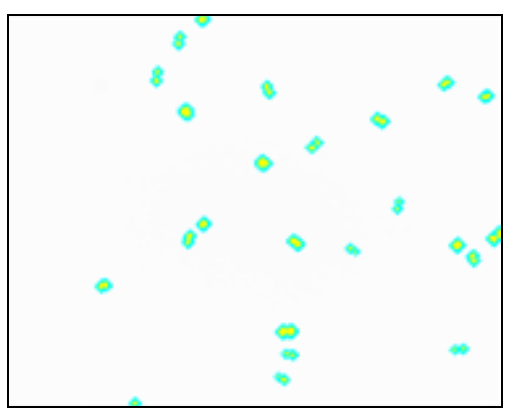

(b) Result of localization for the cells in (a)

Fig. 6. A different type of cell.
Once the cells are localized in the image, the segmentation is performed to extract the cell body using the method presented in Section III. Results are depicted in Figs. 7-8.

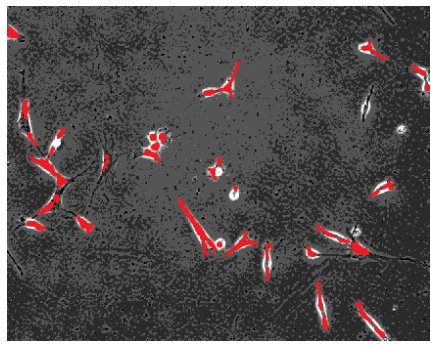

Fig. 7. Segmentation results from Fig. 5.

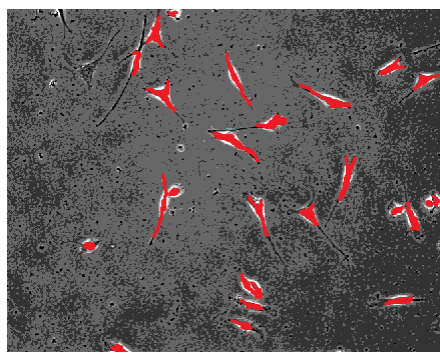

Fig. 8. Segmentation results from Fig. 6.

\section{Spatial Analysis of Detected ObJects}

Within the context of spatial analysis, a relationship between objects means either spatial autocorrelation or randomness. There are several methods in order to model such relationships [20], [21]. In our application, we used grid counting, density plotting, Ripley's K function and pair correlation function to test spatial randomness between the detected cells using the proposed method in Section II.

\section{A. Visual Methods}

The first method used is the grid counting method which is a quadrate-based approach to check for spatial randomness. The method may look simple but it is quite efficient indeed. It works by placing a logical grid on the area where objects lay. This logical grid divides this area into tiles and the number of objects in these tiles is counted.

If the numbers for each tile are close to each other then we can mention about spatial randomness or we can suspect some spatial autocorrelation otherwise. The finer the tile size will mean a smaller number of objects (ideally one object per tile) in a case where random distribution is available.

Figs. 9(a) and 9(b) show the grid count results for the two test sets respectively. From both Figs, some spatial relationship is visible for the test sets.

\begin{tabular}{|c|c|c|c|}
\hline 1 & 0 & 2 & $\infty 4$ \\
\hline 8 & $\circ 5^{\circ}$ & $\infty 3$ & 2 。 \\
\hline $\begin{array}{l}8 \\
2 \\
2\end{array}$ & 0 & $5^{\circ}$ & $\begin{array}{c}\infty_{8}^{\infty} \\
{ }_{8}\end{array}$ \\
\hline 0 & 0 & 0 & 0 \\
\hline
\end{tabular}

(a) Grid count for test set 7826

\begin{tabular}{|c|c|c|c|}
\hline 0 & $8^{8} 8$ & 2 & $\theta^{0}$ \\
\hline 0 & $8^{\circ}$ & 7 & 4 \\
\hline 2 & 2 & 2 & 4 \\
\hline 0 & 3 & 0 & 0 \\
\hline
\end{tabular}

(b) Grid count for test set 8924

Fig. 9. Grid count method for the two test sets. 
A better visualization of the distribution of the objects can be obtained by using a density plot which shows the change in density within the area.

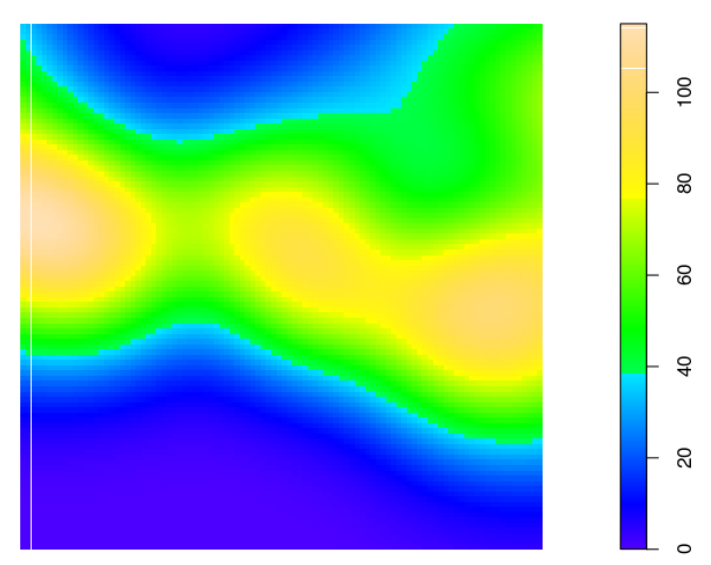

(a) Density plot for test set 7826
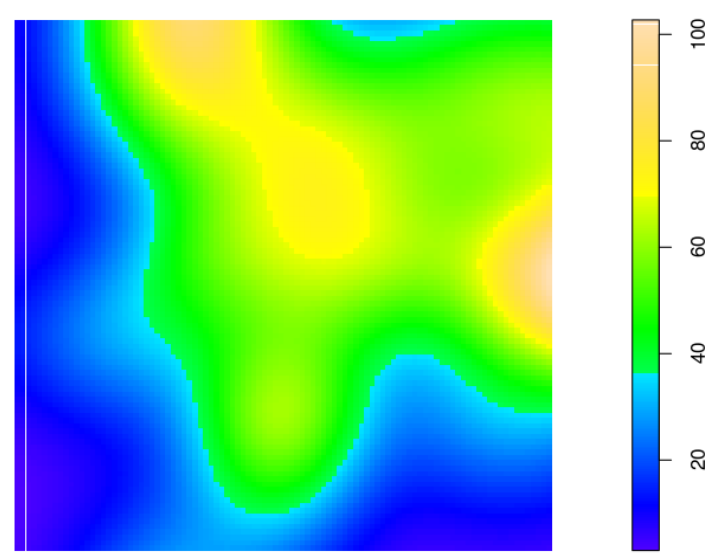

(b) Density plot for test set 8924

Fig. 10. Density plots for the test sets.

Figs. 10(a) and 10(b) show the results for density plots for the datasets. The legends next to the plots show the density measure. Density is visualized by using a colour spectrum ranging from dark blue to bright yellow which indicates increasing density of objects. From both plots, a distribution which can not be interpreted as random is clearly visible.

\section{B. Robust Measures}

Apart from the two methods above, which are aimed for visual inspection of data, there are also more robust statistical measures in order to analyse the distribution of objects within a specific area.

Methods like mean or variance are referred to as first-order properties and do not include or use any information about the area the objects cover. For this reason second-order properties have been introduced [20] in order to take the area coverage and the change [21] in space into account.

An important measure for the second-order properties for a set of objects is the K function proposed by Ripley [22], [23] which works by using the expected number of objects within a specified distance. This expectation is denoted in (1):

$$
\lambda^{-1} E\left[N_{o}(r)\right]
$$

$\lambda$ represents the density of the objects in the area. $N_{o}(r)$ is the number of objects with parameter $r$ denoting the distance around an arbitrary object [20]. This estimation is calculated as shown in (2) [22]:

$$
\hat{K}(r)=\frac{1}{n^{2}}|A| \sum \sum_{i \neq j} \frac{I_{d}\left(d_{i j}\right)}{w_{i j}}
$$

In the equation, $n$ represents the number of objects within the area $|A| . d_{i j}$ denotes the distances between two objects with indices $i$ and $j . I_{d}()$ is a function which indicates the points that will be selected as the objects for the given value of $r$. Finally, $w$ denotes whether edge correction will be applied or not that is some objects that are on the edges on the area may not be accounted for some specific applications [21].

If the objects in the area show complete spatial randomness, then the number of objects within distance $r$ is expected to be:

$$
\hat{K}(r)=\lambda r^{2}
$$

Ripley's $K$ function was applied to the test sets in order to obtain a measure of spatial relation between the cells in the images. Results are presented in Figs. 11(a) and 11(b) respectively:

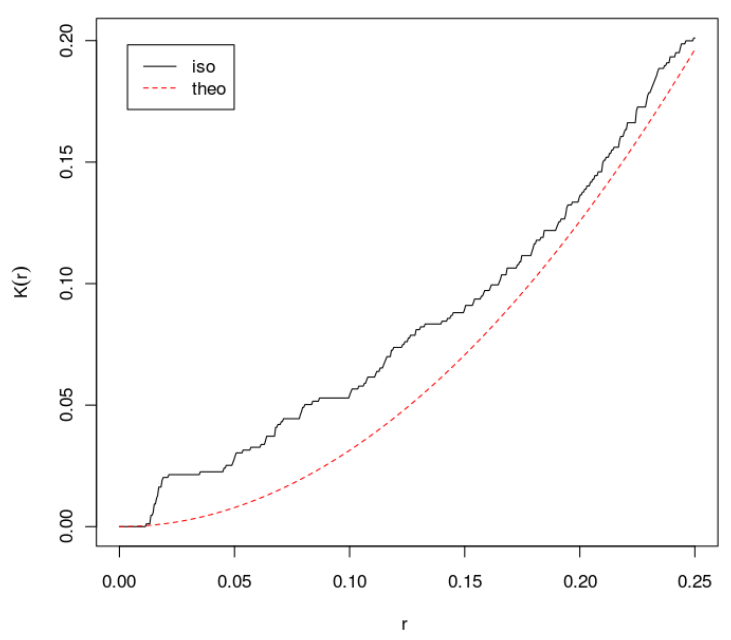

(a) Ripley's $K$ function for test set 7826

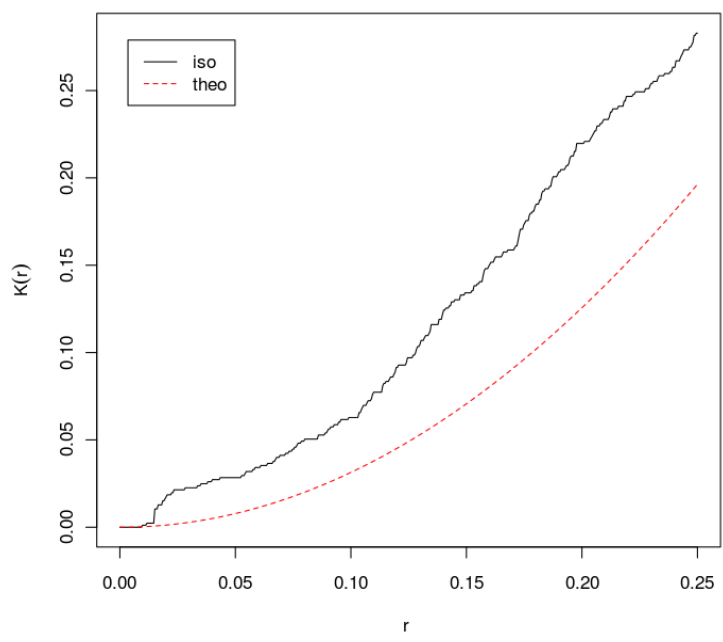

(b) Ripley's $K$ function for test set 8924

Fig. 11. Ripley's $K$ function results. 
The final test was performed using a different second-order property measure named as the pair correlation function [21]. According to the definition of second-order properties given before, this measure also takes the spatial relationship into account just as the Ripley's $K$ function however this method shows the amount of correlation between pairs of objects within a specified distance. For sake of simplicity, we used the definition of the pair correlation function based on the Ripley's $K$ function described in [21] as shown in (4).

$$
g(r)=\frac{1}{d \kappa_{d} r^{d-1}} \frac{d K(r)}{d r}
$$

Which is obviously valid for $\forall r>0$. In the formula, $\kappa_{d}$ is the area for 2-dimensions or volume for 3-dimensions of a unit ball with $d$-dimensions. As the analysis was carried on 2-dimensional objects (found and localized in images), $d=2$ and hence $\kappa_{2}=\Pi$.

The results of the pair correlation function are depicted in Fig. 12. Interestingly, two Figs are quite similar though some minor differences are noticeable. From these Figs, we can see that the objects with a distance less than 0.05 show stronger correlation measures with respect to greater distances.

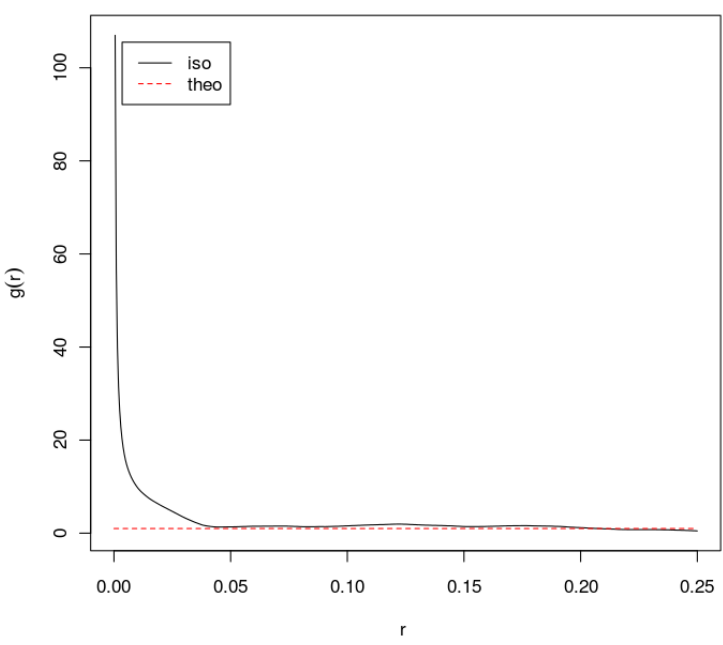

(a) Pair correlation function for test set 7826

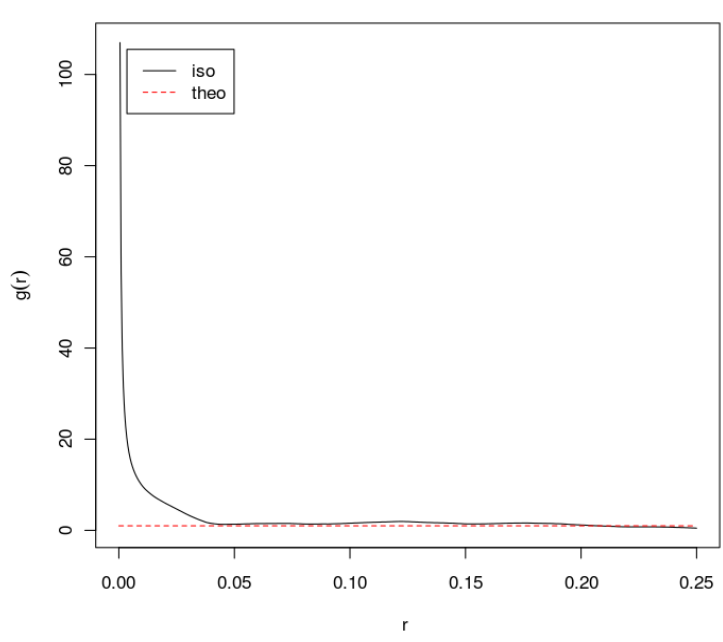

(b) Pair correlation function for test set 8924

Fig. 12. Pair correlation function results for test sets.

\section{CONCLUSIONS AND Future DiRECTIONS}

A novel algorithm for the localization of image objects was proposed in this paper. The method used feature points in order to construct a minimum spanning tree and localize objects using the convex-hull [24] on the individual parts of the generated spanning tree. In addition, a detailed spatial analysis was performed on these localized objects in order to find out spatial relationships.

The methods covered in the paper can be applied to a variety of images including different objects though the experiment was carried out on an image set for fibroblast cells. This experiment carried out here showed that the method worked very accurately using real world data even in case of severe image noise (which can be seen in Figs. 2-6) which is inevitable in any real image but generally more significant in a biological process as there will be other substances or cells in the test environment as well.

The method described here cannot be regarded as a complete segmentation method when compared to [5] and [6]; however, it can be used in the localization process in an easy but effective manner. In addition, the assumption that the objects must be well distinguishable should hold otherwise wrong localization results can be obtained.

Future research will focus on extending this method to a complete segmentation method by combining current approach with a region growing algorithm as in [18]. The authors believe that such a combination will result in better segmentation results which are more robust even in cases of high occlusion, more crowded scenes and severe noise. The spatial analysis carried out here can also be extended to explore more second-order characteristics including the global degree of order [25] which employs the extreme distances in the pair correlation function or following a nearest neighbour approach to model the distribution [17].

\section{REFERENCES}

[1] Y. Rui, T. S. Huang, and S. Chang, "Image Retrieval: Current techniques, promising directions and open issues," Journal of Visual Communication and Image Representation, vol. 10, pp. 39-62, 1999.

[2] P. Scheunders, "A comparison of clustering algorithms applied to color image quantization," Pattern Recognition Letters, vol. 18, pp. 1379-1384, 1997.

[3] A. J. Abrantes and J. S. Marques, "A class of constrained clustering algorithms for object boundary extraction," IEEE Transactions on Image Processing, vol. 5, pp. 1507-1521, 1996.

[4] R. M. Haralick and L. G. Shapiro, "Image segmentation techniques," Computer Vision, Graphics and Image Processing, vol. 29, pp. 100-132, 1985.

[5] A. Blake, C. Rother, M. Brown, P. Perez, and P. Torr, "Interactive image segmentation using an adaptive GMMRF model," Lecture Notes in Computer Science, Springer, vol. 3021, 2004.

[6] J. Fan, D. K. Y. Yau, A. K. Elmagarmid, and W. G. Aref, "Automatic image segmentation by integrating color-edge extraction and seeded region growing," IEEE Transactions on Image Processing, vol. 10, pp. 1454-1466, 2001.

[7] B. W. Silverman, Density Estimation for Statistics and Data Analysis, Chapman and Hall, London, 1986.

[8] J. J. Lennon, "Red-shifts and red herrings in geographical ecology," Ecography, vol. 23, pp. 101-113, 2000.

[9] S. Shekar and H. Xiong, Encyclopedia of GIS, Springer, New York, 2008.

[10] E. Bostanci and B. Bostanci, "A novel method for object localization application to cell localization," in Proc. International Conference on Machine Learning and Computing, Singapore, 2011.

[11] E. Bostanci and B. Bostanci, "Object localization and spatial analysis using computer vision," International Journal of Machine Learning and Computing, vol. 1, 2011. 
[12] S. P. Victor and S. J. Peter, "A novel spanning tree based on clustering algorithm for image mining," European Journal of Scientific Research, vol. 40, pp. 540-546, 2010.

[13] A. S. Tanenbaum, Computer Networks, $4^{\text {th }}$ Ed., Prentice Hall, 2002.

[14] J. B. Kruskal, "On the shortest spanning subtree of a graph and the travelling salesman problem," Proceedings of the American Mathematical Society 7, pp. 48-50, 1956.

[15] R. Prim, "Shortest connection networks and some generalizations," Bell System Technical Journal, vol. 36, pp. 1389-1401, 1957.

[16] E. Rosten and T. Drummond, "Machine learning for high-speed corner detection," in Proceedings of the European Conference on Computer Vision, pp. 430-443, 2006.

[17] S. M. Eckel, "Statistical analysis of spatial point patterns," Ph. D dissertation, Universitat Ulm Institut fur Stochastik, 2008.

[18] S. C. Zhu and A. Yuille, "Region competition: Unifying snakes, region growing and bayes/MDL for multiband image segmentation," IEEE
Transactions on Pattern Analysis and Machine Intelligence, vol. 18, pp. 884-900, 1996.

[19] The Cell Image Library (November, 2012). [Online]. Available: http://cellimagelibrary.org/.

[20] R. S. Bivand, E. J. Pebesma, and V. G. Rubio, Applied Spatial Data Analysis with $R$, Springer, 2008.

[21] M. J. Crawley, The R Book, Wiley-Black Well, 2007.

[22] B. D. Ripley, "The second order analysis of stationary point processes," Journal of Applied Probability, 1976, Number: 13, pp. 255-266.

[23] B. D. Ripley, "Modelling spatial patterns," Journal of the Royal Statistics Society, vol. 39, pp. 172-212, 1977.

[24] W. K. Pratt, Digital Image Processing, 4th edition John Wiley and Sons, Inc., Los Altos, California, 2007.

[25] D. H. Stoyan, Fractals, Random Shapes and Point Fields, J. Wiley and Sons, 1994. 